\title{
The Effect of Tidal Volume on Systemic Inflammation in Acid-Induced Lung Injury
}

\author{
Melissa G. Walker ${ }^{\text {a }}$ Li-Juan Yao ${ }^{\text {a }}$ Eric K. Patterson ${ }^{\text {a }}$ Mariamma G. Joseph ${ }^{\text {b }}$ \\ Gediminas Cepinskas ${ }^{a}$ Ruud A.W. Veldhuizen ${ }^{a, c, d}$ James F. Lewis ${ }^{a, c, d}$
}

Cory M. Yamashita ${ }^{a, c, d}$

a Lawson Health Research Institute, and Departments of ${ }^{\mathrm{b}}$ Pathology, ${ }^{\mathrm{c}}$ Medicine, and ${ }^{\mathrm{d}}$ Physiology and

Pharmacology, University of Western Ontario, London, Ont., Canada

\section{Key Words}

Acute respiratory distress syndrome $\cdot$ Mechanical

ventilation • Isolated perfused mouse lung •

Multiple organ failure

\begin{abstract}
Background: Overwhelming systemic inflammation has been implicated in the progression of acute lung injury (ALI) leading to multiple organ failure (MOF) and death. Previous studies suggest that mechanical ventilation (MV) may be a key mediator of MOF through an upregulation of the systemic inflammatory response. Objectives: It was the aim of this study to investigate mechanisms whereby mechanical stress induced by different tidal volumes may contribute to the development of systemic inflammation and maladaptive peripheral organ responses in the setting of ALI. Methods: An acid aspiration model of ALI was employed in 129X1/SVJ mice through an intratracheal administration of hydrochloric acid followed by MV employing either a low $(5 \mathrm{ml} / \mathrm{kg})$ or high $(12.5 \mathrm{ml} / \mathrm{kg})$ tidal volume ventilation for $120 \mathrm{~min}$. The isolated perfused mouse lung setup was used to assess the specific contribution of the lung to systemic inflammation during MV. Furthermore, lung perfusate collected over the course of MV was used to assess the effects of lung-derived mediators on activation (expression of a proadhesive phenotype) of liver endothelial cells. Results: High tidal volume
\end{abstract}

MV of acid-injured lungs resulted in greater physiologic and histological indices of lung injury compared to control groups. Additionally, there was an immediate and significant release of multiple inflammatory mediators from the lung into the systemic circulation which resulted in greater levels of mRNA adhesion molecule expression in liver endothelial cells in vitro. Conclusions: This study suggests that MV, specifically tidal volume strategy, influences the development of MOF through an upregulation of lung-derived systemic inflammation resulting in maladaptive cellular changes in peripheral organs.

Copyright $\odot 2011$ S. Karger AG, Basel

\section{Introduction}

Acute lung injury (ALI) and its more severe form acute respiratory distress syndrome (ARDS) - represent a spectrum of pulmonary disorders characterized by hypoxemia and reduced lung compliance, in the absence of left-sided heart failure. Despite optimal supportive care of these critically ill patients, mortality due to ALI remains exceedingly high, at approximately 30-40\% [1], with multiple organ failure (MOF) representing the most common cause of death $[2,3]$. The mechanisms leading to the development of non-pulmonary organ failure in this setting remain largely uncharacterized.

Dr. Cory M. Yamashita

Lawson Health Research Institute, Room E5-102

268 Grosvenor St.

London, ON N6A 4V2 (Canada)

E-Mail karger@karger.ch_Accessible online at:

www.karger.com www.karger.com/res 
Currently, the only clinical intervention proven to significantly reduce mortality in patients with ARDS is through the use of low tidal volume mechanical ventilation (MV) [4]. Consistent with this reduction in mortality, patients ventilated with lower tidal volumes had more ventilator-free days, lower levels of systemic inflammatory mediators, and a decreased incidence of non-pulmonary organ failure compared to those ventilated with higher tidal volumes. Although the results of this trial have resulted in widespread adoption of low tidal volume ventilation protocols for patients with ARDS [5], the specific mechanisms linking these strategies to favorable outcomes remain unknown. Therefore, it is critical to understand the mechanisms whereby the use of MV is associated with mortality in ARDS, and to understand how the use of low tidal volume MV in this setting may be beneficial in improving outcomes compared to conventional tidal volume strategies.

The current paradigm for the development of ARDS suggests that multiple and often diverse insults to the lung can result in this syndrome [6]. For example, primary or direct insults, such as gastric acid aspiration and/ or pneumonia, appear to predispose the lung to secondary insults, such as MV, which may then culminate in overwhelming lung inflammation [7]. It is speculated that in this setting, the lung may represent a primary source of signaling mediators which could then translocate into the systemic circulation and elicit pathologic responses in downstream organs $[8,9]$.

The objective of the current study was to specifically examine the effect of different tidal volume strategies within this proposed paradigm of ALI in order to provide insight into potential mechanisms whereby different ventilation strategies could lead to differences in patient outcomes.

\section{Methods}

Study Design

A model of direct lung injury, consisting of acid aspiration in spontaneously breathing mice, was employed with subsequent exposure to MV as a secondary insult. The latter intervention involved supporting the animal with clinically relevant low or high tidal volume MV, using an isolated perfused mouse lung (IPML) setup [10]. This IPML model not only allowed for the analysis of lung-specific effects of MV on lung histology and inflammation, but also for the assessment of inflammatory mediators released from the lung directly into the recirculated perfusate. Subsequent in vitro exposure of liver endothelial cells to perfusate derived from the IPML model provided a means to assess the biological significance of these lung-derived inflammatory mediators in peripheral organ inflammation.

\section{Experimental Groups}

Male 129X1/SVJ mice (Jackson Laboratories, Bar Harbor, Me., USA) were used for the experiments, and all procedures were approved by the Animal Use Subcommittee at the University of Western Ontario. In order to elicit an initial lung injury, an acid aspiration model of ALI in spontaneously breathing animals was employed. Four hours after the initial acid (or air, control) administration, animals were randomized to receive either low (low $\mathrm{V}_{\mathrm{T}}$ group) or high tidal volume (high $\mathrm{V}_{\mathrm{T}}$ group) MV for $120 \mathrm{~min}$ using the IPML model. A total of 32 mice (8/group) were randomized to 1 of 4 experimental conditions: (1) air + low $\mathrm{V}_{\mathrm{T}}$, (2) acid + low $\mathrm{V}_{\mathrm{T}}$, (3) air + high $\mathrm{V}_{\mathrm{T}}$, and (4) acid + high $\mathrm{V}_{\mathrm{T}}$.

\section{Acid Administration}

Mice were anesthetized with an intraperitoneal injection of ketamine (100 mg/kg; Sandoz, Quebec, Que., Canada) and xylazine ( $5 \mathrm{mg} / \mathrm{kg}$; Bayer, Toronto, Ont., Canada). Once adequate anesthesia was achieved, as confirmed by an absence of the toe pinch reflex, mice were positioned dorsally on a rodent stand and intubated with a 20-gauge angiocatheter using the mouse BioLite small animal intubation illumination system (BioTex, Inc., Houston, Tex., USA). Animals randomized to acid administration received $50 \mu \mathrm{l}$ of $0.05 \mathrm{~N} \mathrm{HCl}$ in a drop-wise fashion through the endotracheal tube while the acid was spontaneously inhaled by the animal [11]. Animals randomized to the air group were intubated as described and were permitted to breathe spontaneously for an equal time period. The total duration of the procedure was approximately $3-5 \mathrm{~min}$, at which point animals were extubated and were administered a subcutaneous injection of buprenorphine $(1.5 \mu \mathrm{g})$. Animals were permitted to recover for $4 \mathrm{~h}$ with free access to water and standard chow and were monitored at 15min intervals.

\section{$M V$ Using the IPML Setup}

Four hours following the intratracheal instillation, mice were sacrificed with an intraperitoneal injection of ketamine $(250 \mathrm{mg} /$ $\mathrm{kg})$ and xylazine $(12.5 \mathrm{mg} / \mathrm{kg})$ and were connected to the IPML apparatus as previously described [12]. Briefly, the catheter inserted into the pulmonary artery delivered perfusate (RPMI-1640 lacking phenol red $+2 \%$ low endotoxin grade BSA; Sigma, St. Louis, Mo., USA) into the pulmonary vasculature (at a set flow of $1 \mathrm{ml} / \mathrm{min}$ ), which exited the heart through a second catheter positioned in the left ventricle. The lungs were ventilated using a volume-cycled rodent ventilator (Flexivent, Scireq, Montreal, Que., Canada) with the following ventilation parameters: tidal volume $=5 \mathrm{ml} / \mathrm{kg}$; positive end-expiratory pressure $(\mathrm{PEEP})=$ $3 \mathrm{~cm} \mathrm{H} \mathrm{H}_{2} \mathrm{O}$; respiratory rate $(\mathrm{RR})=60 \mathrm{breaths} / \mathrm{min}$. Once the pulmonary vasculature was cleared of existing blood by visual examination (approx. $12 \mathrm{~min}$ after injection), a 1-ml perfusate sample was obtained (time 0 ) and animals were immediately randomized to low $\mathrm{V}_{\mathrm{T}}$ (tidal volume $=5 \mathrm{ml} / \mathrm{kg}, \mathrm{PEEP}=3 \mathrm{~cm} \mathrm{H}_{2} \mathrm{O}, \mathrm{RR}=$ 60 ) or high $\mathrm{V}_{\mathrm{T}}$ (tidal volume $=12.5 \mathrm{ml} / \mathrm{kg}, \mathrm{PEEP}=3 \mathrm{~cm} \mathrm{H}_{2} \mathrm{O}$, $\mathrm{RR}=30$ ) MV groups. Eleven milliliters of perfusate was then recirculated through the pulmonary vasculature for the duration of MV. A 1-ml sample of perfusate was also obtained at 30, 60, 90 and $120 \mathrm{~min}$ of MV. All perfusate samples were collected and immediately snap frozen in liquid nitrogen and stored at $-80^{\circ} \mathrm{C}$ for subsequent analysis. 
Physiology Measurements

Physiologic parameters including peak inspiratory pressure (PIP) and perfusion pressure were recorded throughout ventilation using Chart version 4.12 software (AD Instruments, Castle Hill, N.S.W., Australia). Immediately following the MV protocol, an automatic pressure-volume perturbation was applied (Flexivent software; Scireq) and a static pulmonary compliance value was calculated using software provided by Scireq.

\section{Lung Lavage, Tissue Fixing and Protein Analysis}

Following 120 min of MV, animals were further randomized into 2 cohorts: (1) the lavage cohort ( $n=5$ /group), in which a full saline lung lavage was performed using $3 \times 1 \mathrm{ml}$ aliquots of $0.15 \mathrm{M} \mathrm{NaCl}$; (2) the histology cohort ( $\mathrm{n}=3$ /group), in which the lungs were excised en bloc and fixed internally at a pressure of 15 $\mathrm{cm} \mathrm{H}_{2} \mathrm{O}$ with $10 \%$ phosphate-buffered formalin (Fisher Scientific, Fair Lawn, N.J., USA) and submersed in $50 \mathrm{ml}$ of $10 \%$ phosphate-buffered formalin, and subsequently, hematoxylin and eosin staining was performed by standardized hospital protocols. Histologic changes were assessed by a lung pathologist who was blinded to the experimental groups. Sections were evaluated by light microscopy for degree and morphologic pattern of injury and was graded semi-quantitatively as follows: 0 , normal; 1 , mild neutrophil influx, edema and hemorrhage; 2 , moderate neutrophil influx, edema and hemorrhage; and 3, marked neutrophil influx, edema and hemorrhage associated with necrosis of bronchiolar epithelium and/or alveoli.

Total Protein and Cytokine and Chemokine Analysis

Total protein was measured in lavage samples as a marker of lung permeability using a Micro BCA protein assay kit (Pierce, Rockford, Ill., USA) following the manufacturer's instructions.

Lavage and perfusate samples were analyzed at each time point for tumor necrosis factor- $\alpha$ (TNF- $\alpha$ ) and interleukin-6 (IL6) using an enzyme-linked immunosorbent assay (ELISA) kit according to the manufacturer's instructions (BD Biosciences, San Diego, Calif., USA).

Perfusate samples obtained at the completion of the MV protocol ( $n=4$ /group) were further analyzed using a Milliplex MAP mouse cytokine/chemokine panel (MPXMCYTO-70K-PMX32; Millipore, Billerica, Mass., USA) for the following 32 analytes: eotaxin, granulocyte colony-stimulating factor (CSF), granulocyte macrophage CSF, interferon- $\gamma$ (IFN- $\gamma$ ), IL-1 $\alpha$, IL-1 $\beta$, IL-2, IL-3, IL-4, IL-5, IL-6, IL-7, IL-9, IL-10, IL-12 (p40), IL-12 (p70), IL-13, IL-15, IL-17, interferon- $\gamma$-induced protein 10 (IP-10), keratinocyte chemoattractant, leukemia inhibitory factor, lipopolysaccharide-induced CXC chemokine, monocyte chemotactic protein 1 , macrophage CSF, monokine induced by IFN- $\gamma$, macrophage inflammatory protein (MIP)- $1 \alpha$, MIP-1 $\beta$, MIP-2, regulated upon activation, normal T cell expressed and secreted (RANTES), TNF- $\alpha$ and vascular endothelial growth factor. Samples were analyzed using the Lumine ${ }^{\circledR} \mathrm{xMAP}^{\circledR}$ detection system on the Luminex $^{100}$ (Linco Research, St. Charles, Mo., USA) according to the manufacturer's instructions.

Mouse Liver Endothelial Cell Culture and Adhesion Molecule Expression

The mouse liver endothelial cells (MLEC) were a kind gift from Dr. Steven Alexander (Louisiana State Health Sciences Center, Shreveport, La., USA). MLEC were cultured in D-valine min- imum essential medium (MEM; PromoCell, Heidelberg, Germany) supplemented with 10\% FBS, 2 mM L-glutamine (Invitrogen, Burlington, Ont., Canada), MEM non-essential amino acids (0.1 mM) (Invitrogen), MEM vitamin mix (1\%) (Invitrogen) and $1 \%$ Pen/Strep.

For the experiments, MLEC were grown in 35-mm Petri dishes until confluence and challenged with $1 \mathrm{ml}$ of lung perfusate from each pool for $2 \mathrm{~h}$. Total RNA was extracted from the cells using Trizol reagent (Invitrogen), as previously described [13]. Three micrograms of total RNA was reverse-transcribed using MMLV Reverse Transcriptase (Invitrogen), following the manufacturer's protocol. Quantitative polymerase chain reaction (qPCR) of adhesion molecules, i.e. intercellular adhesion molecule (ICAM-1), vascular adhesion molecule (VCAM-1) and E-selectin, was performed as described previously [14].

\section{Statistical Analysis}

All data are expressed as the mean \pm SEM. Statistical analysis was performed using SPSS statistical software package for Windows, version 11.0 (SPSS Inc. Chicago, Ill., USA). Treatment effects were determined using a one-way analysis of variance followed by Tukey's post hoc test. Probability $(\mathrm{p})$ values $<0.05$ were considered statistically significant. For analysis of qPCR samples, the baseline fluorescence-subtracted data were analyzed using the 'qPCR' package version 1.2-3 [15] in the software package ' $R$ ' version 2.10.0 (www. r-project.org). The data were fitted to a 5-parameter logistic curve [16] using the Tukey smoothing option to determine Cy0 [17] and the efficiency of the reaction. Clean (nonrecirculated) perfusate control samples were used as the calibrator in each reaction, and the adhesion molecules were normalized to $\beta$-actin expression.

\section{Results}

\section{Lung Physiology}

Prior to the initiation of MV and implementation of the IPML, all animals were carefully observed during the 4 -hour recovery period. There were 3 deaths during this recovery period in animals receiving $\mathrm{HCl}$, and no deaths in animals with air administration. These animals were not included in the statistical analysis.

PIP values measured throughout $120 \mathrm{~min}$ of $\mathrm{MV}$ are shown for animals receiving low tidal volume (fig. 1a) and high tidal volume (fig. 1b). Given the direct relationship between tidal volume and PIP, comparisons are shown between acid- and air-treated animals for the two different ventilation protocols. Independent of ventilation strategy, acid administration resulted in a significant increase in PIP compared to values measured in the airinstilled animals, from the commencement of MV and at all time points throughout the ventilation protocol. There were no significant differences observed in perfusion pressures at any time point between experimental groups within each tidal volume strategy (data not shown). Fig- 
Fig. 1. PIP depicted for air- and acid-instilled mice using low (a) and high tidal volume (b) over the course of MV. There was a significant increase in PIP at all time points during MV in mice with acid administration compared to air-instilled mice, independent of tidal volume strategy. c At completion of MV, there was a significant reduction in lung compliance in acid + high $\mathrm{V}_{\mathrm{T}}$ mice compared to air + high $\mathrm{V}_{\mathrm{T}}$ and acid + low $\mathrm{V}_{\mathrm{T}}$ groups. d Lung lavage protein concentrations measured at the completion of MV demonstrate a significant increase in lavage protein concentrations for mice receiving acid compared to air-administered counterparts ventilated with the same tidal volume. ${ }^{a} \mathrm{p}<0.05$ versus the air group with the same ventilation strategy; ${ }^{\mathrm{b}} \mathrm{p}<0.05$ versus acid + low $\mathrm{V}_{\mathrm{T}}$ mice.

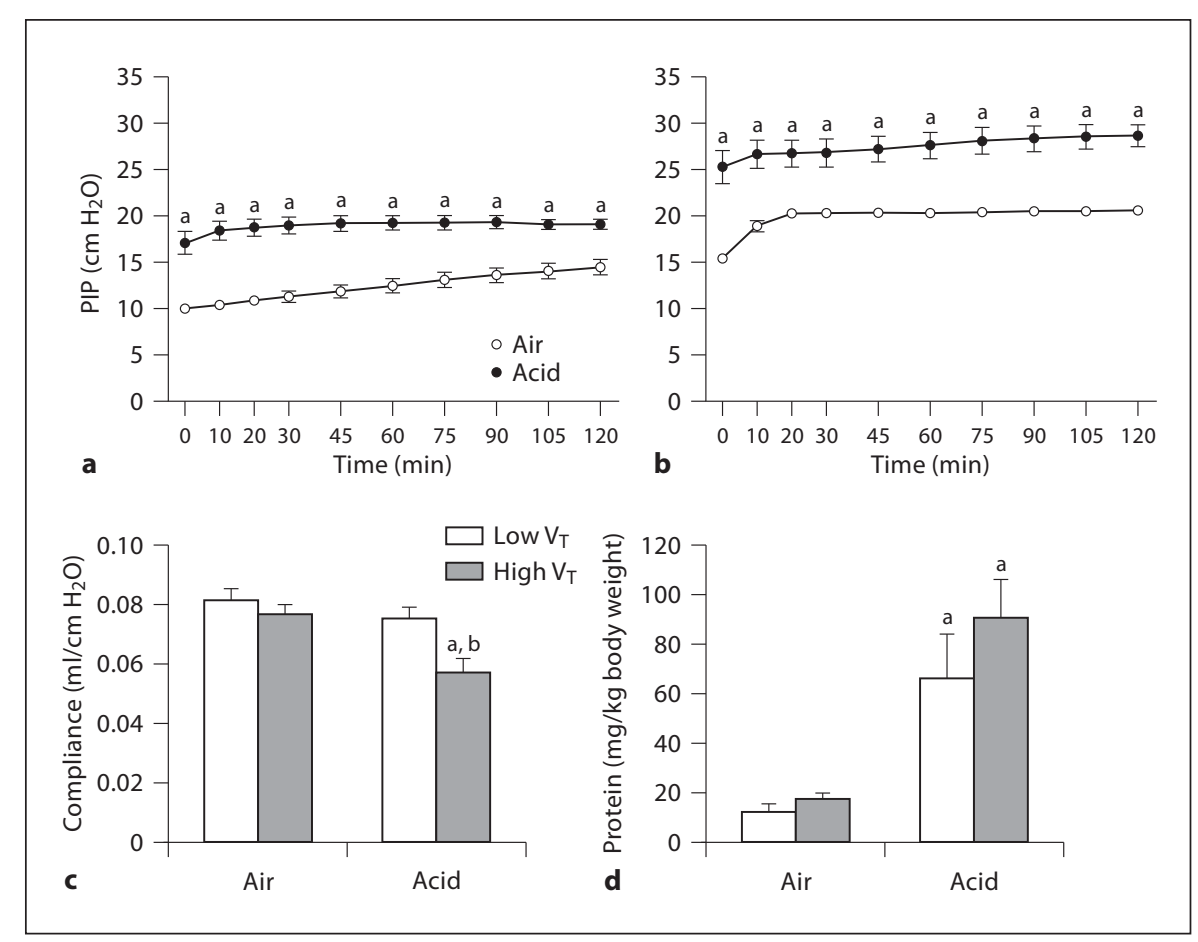

ure 1c shows static pulmonary compliance values obtained at the end of ventilation. There was no significant difference in lung compliance between the air + low $\mathrm{V}_{\mathrm{T}}$ and the air + high $\mathrm{V}_{\mathrm{T}}$ group, and similarly, there was no difference between the air + low $\mathrm{V}_{\mathrm{T}}$ or the acid + low $\mathrm{V}_{\mathrm{T}}$ group. However, acid + high $\mathrm{V}_{\mathrm{T}}$ animals had significantly lower lung compliance at the completion of the ventilation protocol compared to both air + high $\mathrm{V}_{\mathrm{T}}$ and acid + low $\mathrm{V}_{\mathrm{T}}$ groups.

As illustrated in figure 1d, there was no significant effect of tidal volume on lavage protein levels in air-instilled animals (air + low $\mathrm{V}_{\mathrm{T}}$ compared to air + high $\mathrm{V}_{\mathrm{T}}$ group). However, there were significantly higher lavage protein values in mice receiving acid compared to mice receiving air, for both low and high tidal volume MV. In addition, acid + high $\mathrm{V}_{\mathrm{T}}$ animals demonstrated increased protein levels in bronchoalveolar lavage compared to acid + low $\mathrm{V}_{\mathrm{T}}$ animals, although this increase was not statistically significant.

\section{Lung Histology}

The degree and morphologic patterns of injury in the 4 experimental groups are depicted in figure 2 . There were no appreciable differences in lung histology between animals receiving air, irrespective of ventilation strategy, as evidenced by preservation of normal lung architecture and virtual absence of inflammation (fig. 2a, b, normal, grade 0 ). Acid + low $\mathrm{V}_{\mathrm{T}}$ animals demonstrated mild edema, hemorrhage and neutrophilic infiltration compared to animals in the air control (fig. 2c, mild injury, grade 1). Animals in the acid + high $\mathrm{V}_{\mathrm{T}}$ group had more severe lung injury compared to the acid + low $\mathrm{V}_{\mathrm{T}}$ group, as evidenced by severe damage to the bronchial epithelium, pulmonary edema, hemorrhage and abundant neutrophilic infiltration (fig. 2d, severe injury, grade 3). Vascular thrombosis and hyaline membranes were not present in any of these animal groups.

\section{Cytokine Analysis}

Concentrations of IL- 6 and TNF- $\alpha$ in lung lavage at the end of the ventilation period are presented in figure 3 . There was no significant difference in lavage IL-6 levels in animals treated with air instillation irrespective of ventilation strategy. Conversely, there was a significant increase in lavage IL-6 levels in animals in both acid + low $\mathrm{V}_{\mathrm{T}}$ and acid + high $\mathrm{V}_{\mathrm{T}}$ strategies compared to their respective air-instilled control groups. Furthermore, there were significantly higher IL-6 levels in acid + high $\mathrm{V}_{\mathrm{T}}$ compared to acid + low $\mathrm{V}_{\mathrm{T}}$ animals. Although similar trends were observed for lavage TNF- $\alpha$, differences between groups were not statistically significant. 


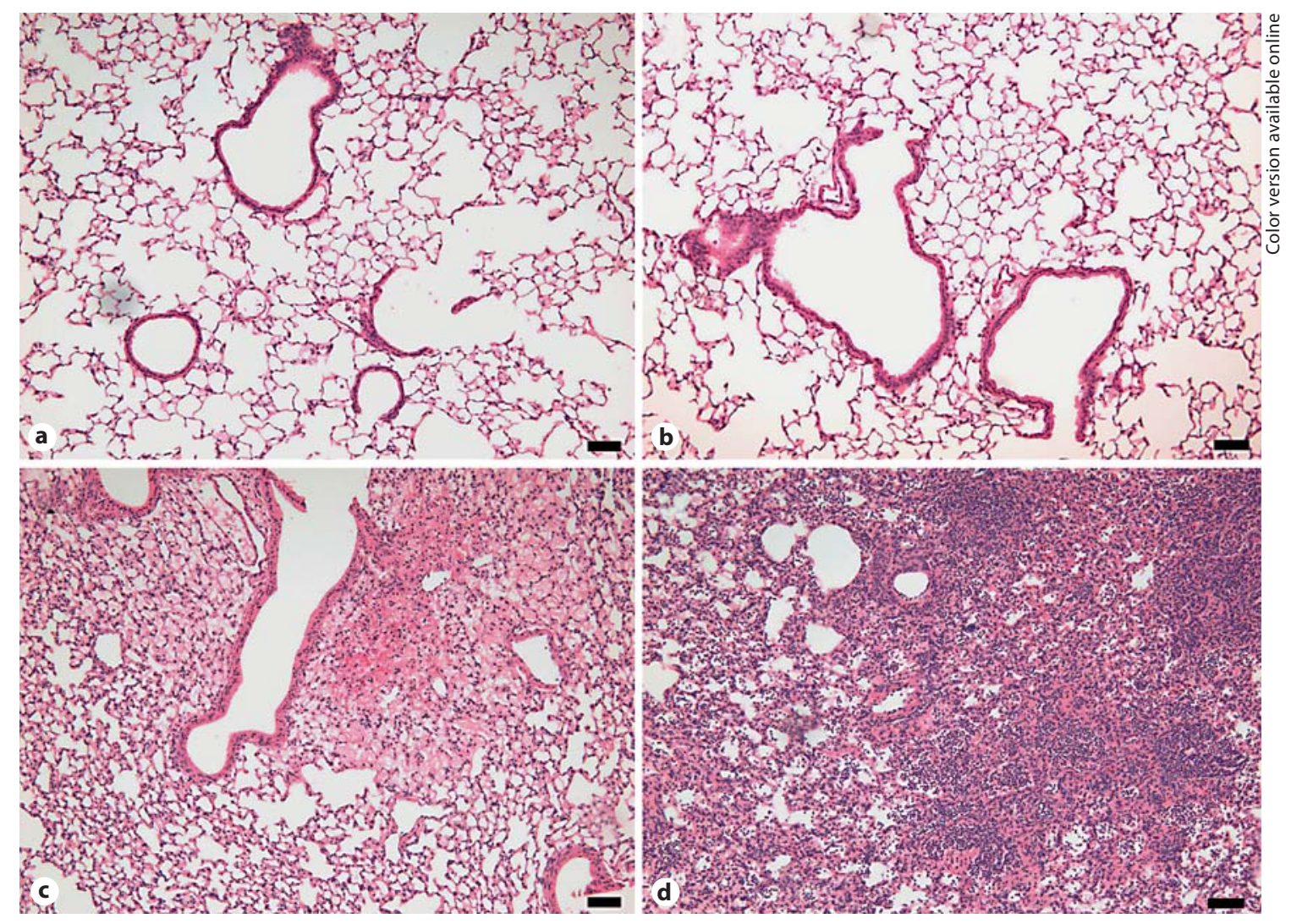

Fig. 2. Histology of hematoxylin and eosin-stained mouse lungs. Air + low $V_{T}$ mice (a), air + high $V_{T}$ mice $(\mathbf{b})$, acid + low $\mathrm{V}_{\mathrm{T}}$ mice $(\mathbf{c})$ and acid + high $\mathrm{V}_{\mathrm{T}}$ mice $(\mathbf{d})$ at the completion of 120 min of mechanical ventilation. $\mathbf{a}$, b Normal airspaces and interstitium. $\times 100$. c Mild injury with patchy edema and mild neutrophil influx in airspaces. $\times 100$. d Severe injury with abundant neutrophil influx in airspaces. Scale bar $=\times 100$.
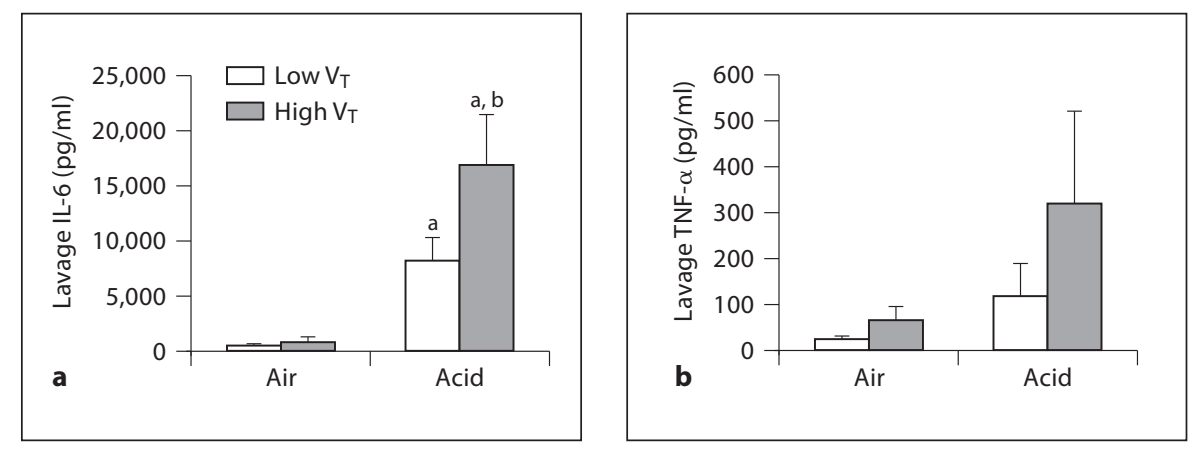

Fig. 3. Lung lavage concentrations of IL-6 (a) and TNF- $\alpha$ (b) measured at the completion of MV demonstrate a significant increase in IL-6 in the acid + low $\mathrm{V}_{\mathrm{T}}$ group compared to the air + low $\mathrm{V}_{\mathrm{T}}$ group, in addition to the acid + high $\mathrm{V}_{\mathrm{T}}$ group compared to the acid + low $V_{T}$ group. Furthermore, there was a significant increase in IL-6 in acid + high $\mathrm{V}_{\mathrm{T}}$ compared with acid + low $\mathrm{V}_{\mathrm{T}}$ mice. There was no significant difference in TNF- $\alpha$ between experimental groups. ${ }^{\mathrm{a}} \mathrm{p}<0.05$ versus the air group with the same ventilation strategy; ${ }^{\mathrm{b}} \mathrm{p}<0.05$ versus acid + low $\mathrm{V}_{\mathrm{T}}$ mice. 


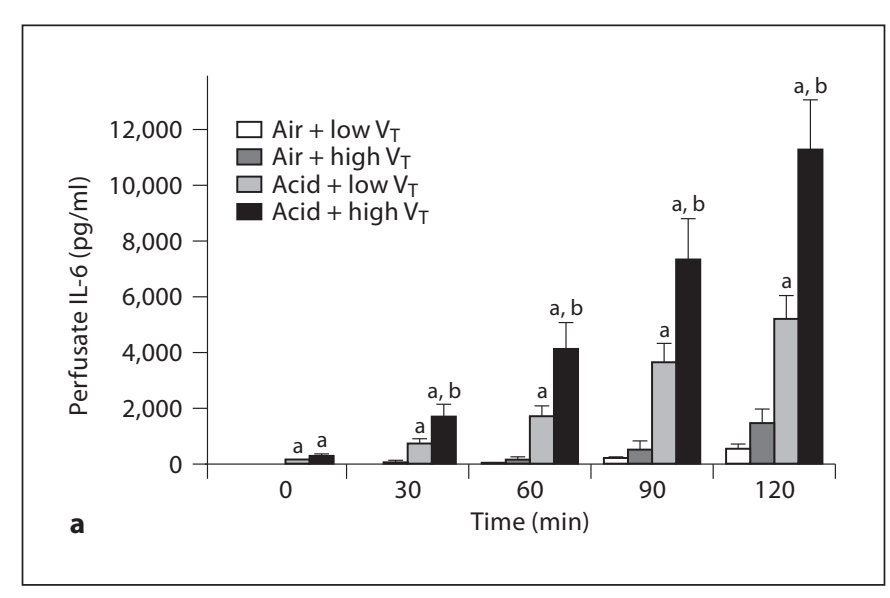

Fig. 4. Lung perfusate concentrations of IL-6 (a) and TNF- $\alpha$ (b) measured throughout the course of MV at 0, 30, 60, 90 and 120 min. At all time points, there was a significant increase in IL-6 levels between acid-instilled groups compared to air-instilled groups ventilated with the same strategy (high or low tidal vol-

Concentrations of IL- 6 and TNF- $\alpha$ measured in perfusate samples obtained at various times over the course of MV are presented in figure 4. At time 0 , immediately after clearing the lung vasculature and prior to the onset of an adoption of a low or high ventilation strategy, there were significantly higher concentrations of IL-6 in acidinjured animals compared to air-instilled controls. Over the course of the ventilation protocol, there were no significant differences in perfusate IL- 6 or TNF- $\alpha$ levels between the air-instilled groups, irrespective of ventilation strategy. Acid + low $\mathrm{V}_{\mathrm{T}}$ animals had significantly higher IL- 6 concentrations compared to Air + low $V_{T}$ animals at the 90- and 120-min time points after the onset of MV. Furthermore, at every time point, animals in the acid + high $\mathrm{V}_{\mathrm{T}}$ group had significantly higher perfusate IL-6 levels compared to both air + high $\mathrm{V}_{\mathrm{T}}$ and acid + low $\mathrm{V}_{\mathrm{T}}$ groups. A similar trend of TNF- $\alpha$ was observed, although the data did not reach statistical significance.

Perfusate collected from experimental groups after completion of MV was further subjected to a multiplex analysis which provided concentrations of 32 cytokines/ chemokines (table 1). There was no statistically significant difference among the experimental groups for IFN$\gamma$, IL-2, IL-7, IL-12 (p40), IL-15, TNF- $\alpha$ and vascular endothelial growth factor (data not shown). Similar to the IL- 6 and TNF- $\alpha$ ELISA data reported above, there was no significant difference in any analyte in animals treated with air, irrespective of ventilation strategy. Acid + low tidal volume resulted in significantly higher concentra-

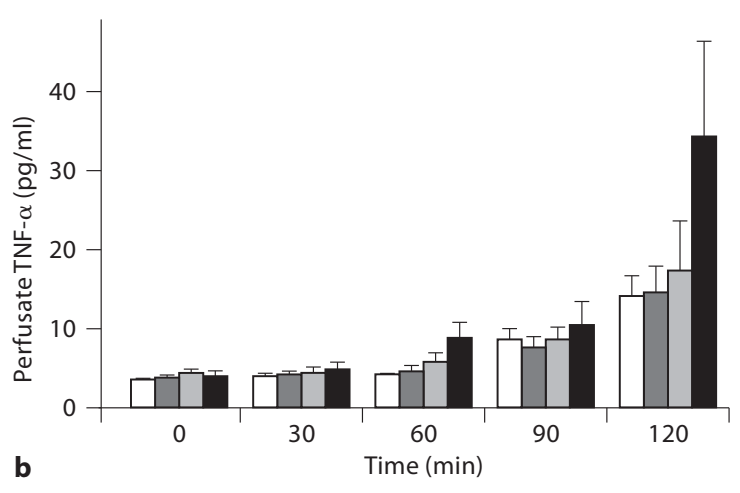

ume). Furthermore, there was a significant increase in IL-6 levels at 30, 60, 90 and $120 \mathrm{~min}$ after the onset of ventilation in acid + high $\mathrm{V}_{\mathrm{T}}$ compared to acid + low $\mathrm{V}_{\mathrm{T}}$ mice. ${ }^{\mathrm{a}} \mathrm{p}<0.05$ versus the air group with the same ventilation strategy; ${ }^{\mathrm{b}} \mathrm{p}<0.05$ versus acid + low $\mathrm{V}_{\mathrm{T}}$ mice.

tions of eotaxin, IL-4, IL-9, IL-12 (p70), IL-13 and IP-10 compared to air + low $\mathrm{V}_{\mathrm{T}}$ animals. Comparing the acid + high $\mathrm{V}_{\mathrm{T}}$ to the air + high $\mathrm{V}_{\mathrm{T}}$ group revealed significantly higher concentrations of 25 of the 32 mediators in acid + high $\mathrm{V}_{\mathrm{T}}$ animals [eotaxin, granulocyte CSF, granulocyte macrophage CSF, IL-1 $\alpha$, IL-1 $\beta$, IL-3, IL-4, IL-5, IL-6, IL9, IP-10, IL-10, IL-12 (p70), IL-13, IL-17, keratinocyte chemoattractant, leukemia inhibitory factor, lipopolysaccharide-induced CXC chemokine, monocyte chemotactic protein 1, macrophage CSF, monokine induced by IFN- $\gamma$, MIP-1 $\alpha$, MIP-1 $\beta$, MIP-2 and RANTES]. Finally, acid + high $\mathrm{V}_{\mathrm{T}}$ animals also had significantly higher concentrations of 11 mediators (eotaxin, IL-1 $\alpha$, IL-1 $\beta$, IL-5, IL-6, IP-10, IL-10, IL-17, M-CSF, MIP-1 $\alpha$, MIP-1 $\beta$ and RANTES) compared to acid + low $\mathrm{V}_{\mathrm{T}}$ animals.

\section{Activation of Liver Endothelial Cells}

In order to assess the biological effects of lung-derived perfusate on peripheral organ function, MLEC were incubated with pooled perfusate from each of the experimental groups. Adhesion molecule mRNA expression was assessed in MLEC after $2 \mathrm{~h}$ of perfusate exposure to reflect the propensity of liver endothelial cells to acquire a 'proadhesive' phenotype ultimately to promote neutrophil sequestration and subsequent peripheral organ dysfunction. Figure 5 depicts adhesion molecule expression in MLEC as a fold increase in VCAM-1, ICAM-1 and Eselectin above control medium (non-circulated perfusate, RPMI $+2 \% \mathrm{BSA}$ ). In pooled perfusate from air-in- 

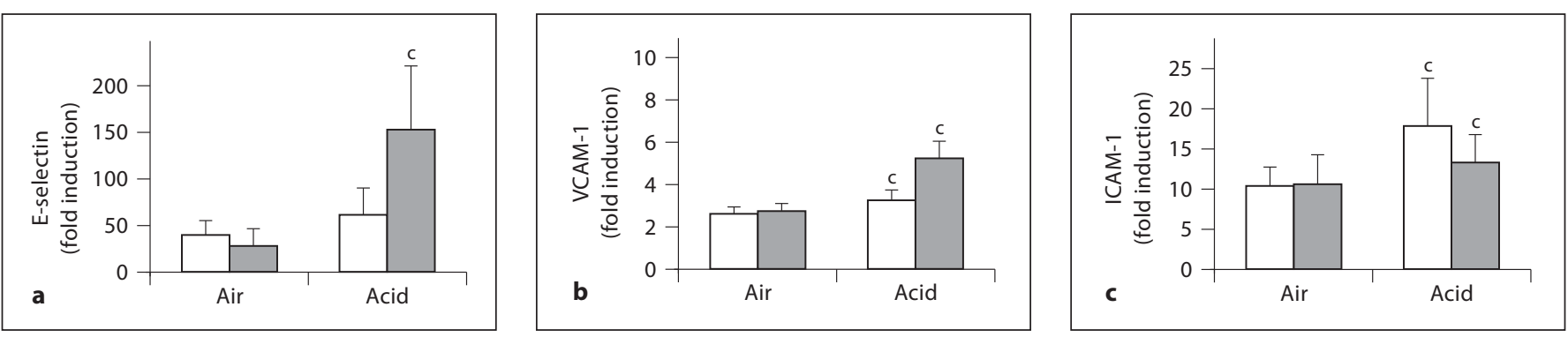

Fig. 5. MLEC adhesion molecule expression for E-selectin (a), VCAM-1 (b) and ICAM-1 (c) represented as fold induction of mRNA compared to control medium (non-circulated perfusate, $\mathrm{RPMI}+2 \% \mathrm{BSA}$ ). Open bars, $\mathrm{Vt}=5 \mathrm{ml} / \mathrm{kg}$; closed bars, $\mathrm{Vt}=12.5$ $\mathrm{ml} / \mathrm{kg}$. There was no significant increase in adhesion molecule mRNA expression in MLEC treated with perfusate from air + low

$\mathrm{V}_{\mathrm{T}}$ or air + high $\mathrm{V}_{\mathrm{T}}$ groups. There was a significant increase in VCAM-1 and ICAM-1 in acid + low $\mathrm{V}_{\mathrm{T}}$ mice compared to control medium and a significant increase in VCAM-1, ICAM-1 and E-selectin in acid + high $\mathrm{V}_{\mathrm{T}}$ mice compared to control medium. ${ }^{c} \mathrm{p}<0.05$ versus control medium.

Table 1. Cytokines/chemokines in perfusate from experimental groups after completion of MV

\begin{tabular}{|c|c|c|c|c|}
\hline $\begin{array}{l}\text { Mediator } \\
\mathrm{pg} / \mathrm{ml}\end{array}$ & \multicolumn{2}{|l|}{ Air } & \multicolumn{2}{|l|}{ Acid } \\
\hline G-CSF & $102.27 \pm 61.55$ & $265.43 \pm 176.05$ & $420.63 \pm 129.00$ & $2,164.09 \pm 892.38^{\mathrm{a}}$ \\
\hline GM-CSF & $4.13 \pm 2.31$ & $14.14 \pm 5.77$ & $36.58 \pm 5.99$ & $78.39 \pm 23.56^{\mathrm{a}}$ \\
\hline IL- $1 \alpha$ & $18.56 \pm 7.11$ & $34.04 \pm 17.42$ & $55.97 \pm 12.48$ & $149.72 \pm 37.92^{\mathrm{a}, \mathrm{b}}$ \\
\hline IL-4 & $0.12 \pm 0.12$ & $0.54 \pm 0.14$ & $0.82 \pm 0.05^{\mathrm{a}}$ & $1.16 \pm 0.18^{\mathrm{a}}$ \\
\hline IL-5 & $0.10 \pm 0.10$ & $0.77 \pm 0.70$ & $1.02 \pm 0.34$ & $3.07 \pm 0.80^{\mathrm{a}, \mathrm{b}}$ \\
\hline IL-6 & $762.2 \pm 317$ & $1,591 \pm 959$ & $5,013 \pm 936$ & $12,093 \pm 2,551^{\mathrm{a}, \mathrm{b}}$ \\
\hline IL-9 & 7.74 & $12.5 \pm 7.65$ & $31.0 \pm 5.45^{\mathrm{a}}$ & $48.82 \pm 8.7^{\mathrm{a}}$ \\
\hline IP-10 & $96.7 \pm 16.8$ & $122.25 \pm 30$ & $266.4 \pm 48.7^{\mathrm{a}}$ & $477.17 \pm 59.8^{\mathrm{a}, \mathrm{b}}$ \\
\hline $\mathrm{KC}$ & $959.7 \pm 495.1$ & $1,133.6 \pm 529.5$ & $2,297.5 \pm 499$ & $4,968.9 \pm 1,327.9^{a}$ \\
\hline LIF & $0.20 \pm 0.20$ & $2.31 \pm 2.12$ & $5.54 \pm 1.43$ & $20.50 \pm 7.8^{\mathrm{a}}$ \\
\hline LIX & $53.01 \pm 13.1$ & $71.45 \pm 31.9$ & $131.38 \pm 26.3$ & $616 \pm 238^{a}$ \\
\hline MCP-1 & $8.85 \pm 4.32$ & $24.0 \pm 15.8$ & $52.9 \pm 11.9$ & $138.4 \pm 47.5^{\mathrm{a}}$ \\
\hline M-CSF & $0.99 \pm 0.825$ & $1.50 \pm 0.78$ & $2.61 \pm 0.92$ & $7.23 \pm 0.61^{\mathrm{a}, \mathrm{b}}$ \\
\hline MIG & $8.64 \pm 4.8$ & $6.03 \pm 2.72$ & $10.44 \pm 2.5$ & $40.12 \pm 16.1^{\mathrm{a}}$ \\
\hline MIP-1 $\alpha$ & $79.6 \pm 16.8$ & $62.3 \pm 15.9$ & $87.12 \pm 26.9$ & $340.7 \pm 78.9^{\mathrm{a}, \mathrm{b}}$ \\
\hline MIP-1 $\beta$ & $101.8 \pm 21.6$ & $83.84 \pm 21.5$ & $118.9 \pm 41.2$ & $443.4 \pm 81.1^{\mathrm{a}, \mathrm{b}}$ \\
\hline MIP-2 & $660.9 \pm 183.0$ & $557 \pm 246$ & $1,274.3 \pm 272.6$ & $6,377.8 \pm 2,733.9^{a}$ \\
\hline RANTES & $2.80 \pm 0.58$ & $3.39 \pm 1.29$ & $5.09 \pm 0.86$ & $13.12 \pm 1.67^{\mathrm{a}, \mathrm{b}}$ \\
\hline
\end{tabular}

G-CSF = Granulocyte CSF; GM-CSF = granulocyte macrophage CSF; IP-10 = interferon- $\gamma$-induced protein 10 ; KC = keratinocyte chemoattractant; LIF = leukemia inhibitory factor; LIX = liposaccharide-induced CXC chemokine; MCP-1 = monocyte chemotactic protein $1 ; \mathrm{M}-\mathrm{CSF}=$ macrophage CSF; MIG = monokine induced by IFN $-\gamma ; \mathrm{MIP}=$ macrophage inflammatory protein. ${ }^{\mathrm{a}} \mathrm{p}<0.05$ versus air group with the same ventilation strategy; ${ }^{b} \mathrm{p}<0.05$ versus acid + low $\mathrm{V}_{\mathrm{T}}(\mathrm{n}=4$ /group). 
stilled mice, there was no significant effect of tidal volume strategy on the expression of VCAM-1, ICAM-1 or E-selectin mRNA expression although both groups demonstrated an increase in adhesion molecule mRNA above control medium. MLEC cultured with pooled perfusate derived from the acid + low $\mathrm{V}_{\mathrm{T}}$ animals resulted in an increased expression of both ICAM-1 and VCAM-1 compared to uncirculated perfusate, while MLEC cultured in the presence of perfusate from acid + high $\mathrm{V}_{\mathrm{T}}$ animals resulted in significant increased expression of all three adhesion molecules compared to uncirculated perfusate.

\section{Discussion}

The overall objective of this study was to investigate potential mechanisms responsible for differences in mortality and ultimately MOF with the use of low versus high tidal volume MV. Specifically, we have shown in our model of ALI that differences in MV strategies in the context of a pre-existing lung injury had a marked effect on the inflammatory response within the lung, which in turn, was associated with a rapid release of potentially injurious mediators into the circulation shortly after the onset of MV. Furthermore, this material released by the injured lung in response to MV had a detrimental impact on peripheral organ endothelial cell phenotype. Based on this study, we conclude that inflammatory mediators released from the lung into the systemic circulation, in this case specifically due to the use of higher tidal volumes, may represent one potential mechanism which may account for the high prevalence of MOF associated with ARDS.

The experimental models employed in the current study reflect a common clinical scenario of gastric aspiration followed by MV resulting in an ALI-like picture [18, 19]. The secondary insult of MV was employed using the IPML procedure which allowed evaluation of the effects of clinically relevant ventilation strategies on our various outcomes. Although using the IPML model failed to account for the multiple, complex interactions which may occur in an intact systemic circulation in vivo, elimination of the circulatory effects enabled direct observation of the contribution of the lungs toward systemic inflammation. Moreover, the ability to sample lung-derived perfusate throughout the course of MV with the IPML technique not only offered insight into the time-dependent release of mediators into the systemic circulation, but also allowed us to study peripheral organ effects of the lungderived mediators obtained from the various groups.
Although the impact of MV on proinflammatory mediator release into the airspace has been well established, and has been termed 'biotrauma', its direct contribution to systemic inflammation and peripheral organ dysfunction remains unclear $[20,21]$. Clinically, the use of higher tidal volumes (i.e. $12 \mathrm{ml} / \mathrm{kg}$ ) was associated with significantly greater systemic inflammation and organ failure in patients with ALI [4]. Our studies confirm that the stress induced by high tidal volume MV of an injured lung resulted in a clear amplification of both the pulmonary and systemic inflammatory response, the latter resulting from a translocation of mediators into the circulation from the lung almost immediately after the onset of MV. Thus, we believe that the transition from pulmonary to systemic inflammation can be mediated directly by the adopted ventilation strategy, and this represents a potential mechanism or component of the paradigm in ALI and MOF by which high tidal volume MV may affect mortality in patients with ARDS.

In addition to this general conclusion, several aspects of the current study provided insight into the specific effects of MV on the progression of ALI. Firstly, we observed a significant release of inflammatory mediators occurring almost immediately after the onset of high tidal volume MV of the injured lung. Although the use of low tidal volumes is currently accepted as the gold standard of ventilatory care in patients with ALI, it is currently only indicated after the diagnosis of ALI has been well established. In many cases, conventional or at least higher tidal volumes are employed for an undefined period of time prior to the onset of clinically apparent disease. Therefore, we speculate that significant systemic mediator release may occur in patients with lung injury well before the clinical criteria for ALI are met, likely resulting in 'sub-clinical' systemic inflammation before the use of low $\mathrm{V}_{\mathrm{T}}$ strategies are implemented. This window of time may represent a critical time frame during which an injured or inflamed lung may be particularly susceptible to the effects of higher tidal volume MV or other secondary insults placing patients at high risk of developing ALI.

Another important observation in the current study was the marked increase in several different inflammatory mediators both in the lung lavage and perfusate within $2 \mathrm{~h}$ of MV. This finding underscores the complexity of the host's generalized inflammatory response observed in the setting of lung injury. For example, in the current study, 25 out of the 32 inflammation-relevant mediators measured were significantly elevated in the acid + high $\mathrm{V}_{\mathrm{T}}$ cohort of animals compared to the air + 
high $\mathrm{V}_{\mathrm{T}}$ cohort of animals. This diverse range of mediator release observed in this relatively straightforward, controlled model of ALI underscores the notion that a far greater number of clinical variables undoubtedly lead to complex interactions in human subjects with ARDS [22]. This particular observation, although perhaps intuitive, likely accounts for the lack of efficacy of previous molecular-specific therapies that have been evaluated for ARDS, as the systemic effect of lung-derived mediators may represent an aggregate or compound effect [23]. Related to these clinical observations are recent in vitro studies involving a lipopolysaccharide model of ALI in which neutralization of individual specific mediators (TNF- $\alpha$ and IL-6) within perfusate samples did not prevent expression of a proinflammatory phenotype in liver endothelial cells. On the other hand, the biological activity of this perfusate was largely minimized upon global cytokine degradation via heating [14]. These data suggest that it is likely not the action of one mediator, but the complex interactions of many mediators that contribute to the development of MOF.

Finally, it is important to note that the current study employed tidal volume indices that are used routinely in clinical practice [24]. Tidal volumes used in the non-injured lungs did not impact any of the measured outcomes and may suggest that only an inflamed or injured lung may be susceptible to this second hit injury stimulus. However, in the context of a pre-existing injury induced by $\mathrm{HCl}$, even acid + low tidal volume, resulted in significant pulmonary, systemic inflammation with proinflammatory effects on liver endothelial cells with an effect that was further augmented with the use of higher tidal volumes. The significant release of inflammatory mediators in lung-injured animals ventilated with low tidal volumes underscores the notion that despite the current 'gold standard' care of lung-protective strategies in patients with ARDS, there exists a substantial window of opportunity to further improve systemic inflammation with additional therapies and to ultimately reduce the inci- dence of MOF. In this regard, further optimization of ventilation strategies through the use of high-frequency oscillation, for example, or the administration of additional lung-based therapies may represent further advances which may lead to improved patient outcomes. The models employed in this paper may provide an ideal modeling paradigm which could be used to evaluate and identify proposed treatment options and to determine whether in fact they will be successful in minimizing systemic inflammation and ultimately peripheral organ dysfunction.

In conclusion, the current study offers potential mechanisms whereby clinical trials have shown a significant decrease in the mortality of patients with ARDS when ventilating with lower tidal volumes [4]. We believe that our studies have expanded on what is known (based on previous literature) by demonstrating that in addition to the significant effects on lung physiology and inflammation, low tidal volume ventilation reduced systemic inflammation and ultimately resulted in a decrease in the biological effects on liver endothelial cells. The immediate clinical ramifications of these findings implicate the initiation of ventilation as a potentially crucial window of opportunity to intervene in the natural progression of this disease. Specifically, limiting the release of inflammatory mediators secondary to onset of MV may represent a key therapeutic window in the ARDS/MOF paradigm where lung-specific interventions may ultimately influence the eventual outcomes in these patients.

\section{Acknowledgements}

The authors would like to thank Dr. Kelly Summers and Ms. Shannon Mifflin for their assistance with cytokine analysis and Lynda McCaig for her technical assistance with the IPML model.

This study was supported by funds provided by the Canadian Institutes of Health Research (MOP11666), the Lawson Health Research Institute and the Department of Medicine at the University of Western Ontario.
References

Lung and Systemic Effects of Tidal

Volume Strategy
1 Zambon M, Vincent JL: Mortality rates for patients with acute lung injury/ARDS have decreased over time. Chest 2008;133:11201127.

-2 Stapleton RD, Wang BM, Hudson LD, Rubenfeld GD, Caldwell ES, Steinberg KP: Causes and timing of death in patients with ARDS. Chest 2005;128:525-532.
3 Montgomery AB, Stager MA, Carrico CJ, Hudson LD: Causes of mortality in patients with the adult respiratory distress syndrome. Am Rev Respir Dis 1985;132:485-489.

4 Ventilation with lower tidal volumes as compared with traditional tidal volumes for acute lung injury and the acute respiratory distress syndrome. The Acute Respiratory Distress Syndrome Network. N Engl J Med 2000;342:1301-1308. 
5 Kallet RH, Jasmer RM, Pittet JF, Tang JF, Campbell AR, Dicker R, Hemphill C, Luce JM: Clinical implementation of the ARDS network protocol is associated with reduced hospital mortality compared with historical controls. Crit Care Med 2005;33:925-929.

6 Bosma KJ, Lewis JF: Emerging therapies for treatment of acute lung injury and acute respiratory distress syndrome. Expert Opin Emerg Drugs 2007;12:461-477.

$\checkmark 7$ Moore FA, Moore EE, Read RA: Postinjury multiple organ failure: role of extrathoracic injury and sepsis in adult respiratory distress syndrome. New Horiz 1993;1:538-549.

-8 Jiang JS, Chou HC, Wang LF, Lang YD, Chen CM: Effects of activated protein $\mathrm{C}$ on ventilator-induced lung injury in rats. Respiration 2010;80:246-253.

-9 Ranieri VM, Suter PM, Tortorella C, De TR, Dayer JM, Brienza A, Bruno F, Slutsky AS: Effect of mechanical ventilation on inflammatory mediators in patients with acute respiratory distress syndrome: a randomized controlled trial. JAMA 1999;282:54-61.

10 Hite RD, Morris PE: Acute respiratory distress syndrome: pharmacological treatment options in development. Drugs 2001;61:897907.

-11 Nagase T, Uozumi N, Ishii S, Kume K, Izumi T, Ouchi Y, Shimizu T: Acute lung injury by sepsis and acid aspiration: a key role for $\mathrm{cy}$ tosolic phospholipase A2. Nat Immunol 2000;1:42-46.
12 Walker MG, Tessolini JM, McCaig L, Yao LJ, Lewis JF, Veldhuizen RA: Elevated endogenous surfactant reduces inflammation in an acute lung injury model. Exp Lung Res 2009; 35:591-604.

13 Suzuki T, Yamashita C, Zemans RL, Briones N, Van LA, Downey GP: Leukocyte elastase induces lung epithelial apoptosis via a PAR1, NF- $\{$ kappa $\}$ B, and p53-dependent pathway. Am J Respir Cell Mol Biol 2009;41:742755.

14 Markovic N, McCaig LA, Stephen J, Mizuguchi S, Veldhuizen RA, Lewis JF, Cepinskas G: Mediators released from LPS-challenged lungs induce inflammatory responses in liver vascular endothelial cells and neutrophilic leukocytes. Am J Physiol Gastrointest Liver Physiol 2009;297:G1066-G1076.

15 Ritz C, Spiess AN: qpcR: an R package for sigmoidal model selection in quantitative real-time polymerase chain reaction analysis. Bioinformatics 2008;24:1549-1551.

16 Spiess AN, Feig C, Ritz C: Highly accurate sigmoidal fitting of real-time PCR data by introducing a parameter for asymmetry. BMC Bioinformatics 2008;29:221.

17 Guescini M, Sisti D, Rocchi MB, Stocchi L, Stocchi V: A new real-time PCR method to overcome significant quantitative inaccuracy due to slight amplification inhibition. BMC Bioinformatics 2008;9:326.
8 Hudson LD, Steinberg KP: Epidemiology of acute lung injury and ARDS. Chest 1999;116: $74 \mathrm{~S}-82 \mathrm{~S}$.

19 Livingston DH, Mosenthal AC, Deitch EA: Sepsis and multiple organ dysfunction syndrome: a clinical-mechanistic overview. New Horiz 1995;3:257-266.

20 dos Santos CC, Slutsky AS: The contribution of biophysical lung injury to the development of biotrauma. Annu Rev Physiol 2006; 68:585-618.

21 Tremblay LN, Slutsky AS: Ventilator-induced injury: from barotrauma to biotrauma. Proc Assoc Am Physicians 1998;110: 482-488.

22 Ware LB, Matthay MA: The acute respiratory distress syndrome. N Engl J Med 2000; 342:1334-1349.

23 Rubenfeld GD, Caldwell E, Peabody E, Weaver J, Martin DP, Neff M, Stern EJ, Hudson LD: Incidence and outcomes of acute lung injury. N Engl J Med 2005;353:16851693.

24 Young MP, Manning HL, Wilson DL, Mette SA, Riker RR, Leiter JC, Liu SK, Bates JT, Parsons PE: Ventilation of patients with acute lung injury and acute respiratory distress syndrome: has new evidence changed clinical practice? Crit Care Med 2004;32:12601265. 